\title{
Reduced magnetohydrodynamics and parallel spectral transfer
}

\author{
S. Oughton \\ Department of Mathematics, University of Waikato, Private Bag 3105, Hamilton, New Zealand \\ P. Dmitruk and W. H. Matthaeus \\ Bartol Research Institute, University of Delaware, Newark, Delaware 19716
}

(Received 7 January 2004; accepted 20 February 2004; published online 16 April 2004)

\begin{abstract}
The self-consistency of the reduced magnetohydrodynamics (RMHD) model is explored by examining whether (parallel) spectral transfer might invalidate the assumptions employed in deriving it. Using direct numerical simulations we find that transfer of energy to structures with high parallel wavenumber is in fact limited by ongoing perpendicular transfer. Thus, the dynamics associated with RMHD models remains consistent with the underlying assumptions of RMHD. In particular, in well-resolved simulations it is neither necessary nor correct to introduce additional dissipation terms that (artificially) damp spectral transfer parallel to the mean magnetic field $\mathbf{B}_{0}$. ㄷ) 2004 American Institute of Physics. [DOI: 10.1063/1.1705652]
\end{abstract}

\section{INTRODUCTION}

The equations of reduced magnetohydrodynamics (RMHD) are a nonlinear approximation to the equations of full (i.e., nonlinear, compressible, and three-dimensional) MHD, appropriate in certain situations where there is a strong mean magnetic field $\mathbf{B}_{0}$. Physically, systems governed by the RMHD equations can be considered as a set of planes of two-dimensional (2D) MHD fluctuations, which are coupled together by long wavelength Alfvén waves, propagating normal to the planes. First derived by Kadomtsev and Pogutse, ${ }^{1}$ they have been rederived from various other perspectives several times. ${ }^{2-6}$ In particular, in Montgomery's ${ }^{3}$ derivation the stated motivation was to find a set of equations which simultaneously retained nonlinearities while remaining of $O(1)$ timescale in the limit as $B_{0} \rightarrow \infty$; i.e., somehow avoiding the large-magnitude time derivatives associated with Alfvén waves in this limit. Subsequently, RMHD models have been widely used in both terrestrial and space physics applications, including plasma fusion studies ${ }^{1,5}$ and coronal heating models (see, e.g., Refs. 7 and 8 and references therein).

In this paper we focus on several apparently underappreciated features of the RMHD approximation, associated with the nature of spectral transfer in the direction of $\mathbf{B}_{0}$. In particular, we show that for fluctuations evolving according to the RMHD equations, parallel spectral transfer is selflimiting - despite the absence of dissipation in this direction (see also Ref. 9).

An important feature of RMHD systems is that highfrequency Alfvén waves are-by construction-excluded, in effect by the insistence that the "wave terms" are (at most) comparable in magnitude to the nonlinear ones. This leads to a compact representation, which, as the applied magnetic field becomes very strong, lacks fast timescales and small parallel lengthscales. The RMHD approximation also leads to the dissipative terms becoming independent of the parallel gradients, e.g., viscous dissipation is proportional to $\nabla_{\perp}^{2} \mathbf{v}$ rather than the full Laplacian of the velocity.
However, these features of RMHD also raise concerns about the self-consistency of its derivation. A question that arises is whether the conditions that are imposed in deriving RMHD will remain valid as the fields evolve. For example, there are no explicit factors in the RMHD model that prevent spectral cascade of a type that would steepen gradients in the direction parallel to $\mathbf{B}_{0}$. Also, as noted above, RMHD has no dissipation in the parallel direction. Consequently, lacking both restrictions on the (parallel) cascade and parallel dissipation effects-either of which might limit the formation of structure with ever finer parallel length scales-it seems possible that such high $k_{\|}$excitations could emerge dynamically in solutions of the RMHD equations. This technical issue leads to concerns that RMHD invalidates itself, and therefore might question the usefulness of the model. Here we examine the issue directly using numerical simulations. We will conclude that parallel spectral transfer is indeed limited-not by dissipation-but by the efficacy of perpendicular spectral transfer. Thus, the RMHD model can remain valid without use of artificial dissipation terms that damp fluctuations with high $k_{\|}$.

As is clear from the derivation of the RMHD equations, the couplings retained in an RMHD model are a subset of those present in the full MHD model. This justifies at least partially the use of RMHD models by some authors ${ }^{9-12}$ to investigate theoretical issues in general MHD theory. By investigating in some detail the structure of RMHD in the context of full MHD, we will provide here a context for understanding the generality and limitations of such conclusions.

The paper is structured as follows. Section II reviews the nature of the RMHD approximation. Discussion of related points regarding cascades-in both full and reduced MHD-is given in Sec. III. Simulation results are presented and discussed in Sec. IV, for the case of periodic boundary conditions. A conclusions section and several Appendices close the paper. 


\section{THE RMHD APPROXIMATION}

The usual primary assumption of RMHD is that a strong uniform magnetic field, $\mathbf{B}_{0}=B_{0} \hat{\mathbf{z}}$, is present. ${ }^{1,3,5,6}$ By strong, one means that fluctuations in the velocity and magnetic fields have much smaller energy densities than that associated with $B_{0}$. Insisting further that the nonlinear terms (e.g., $\mathbf{b} \cdot \nabla \mathbf{v}$ ) be comparable in size to the linear ones (e.g., $\left.\mathbf{B}_{0} \cdot \nabla \mathbf{v}\right)$, means that it is necessary to restrict gradients along $\mathbf{B}_{0}$ to be small. This leads to a natural rescaling of (i) the parallel coordinate, $z$, to be purely large-scale and (ii) the mean field strength $B_{0}$, which becomes $O(1)$. We denote the rescaled field strength as $B_{0}^{\prime}$, with $B_{0}$ the strength of the actual physical field. Similarly, $z^{\prime}$ is the rescaled version of physical coordinate $z$. For consistency, we denote the variable which is Fourier conjugate to $z^{\prime}$ by $k_{z}^{\prime}$.

The well-known leading-order equations which result from this approximation are referred to as the equations of RMHD. They involve an incompressible zero-mean planepolarized velocity $\mathbf{v}\left(x, y, z^{\prime}, t\right)=\left(v_{x}, v_{y}, 0\right)$ and a similarly plane-polarized magnetic fluctuation. In nondimensionalized (Alfvén speed) units they can be expressed ${ }^{3,6}$ as equations for the evolution of the fluctuations in the fluid vorticity $\omega$ and the magnetic vector potential $a$ :

$$
\begin{aligned}
& \left(\frac{\partial}{\partial t}+\mathbf{v} \cdot \nabla\right) \omega=\mathbf{b} \cdot \nabla_{\perp} j+\nu \nabla_{\perp}^{2} \omega+B_{0}^{\prime} \frac{\partial j}{\partial z^{\prime}}, \\
& \left(\frac{\partial}{\partial t}+\mathbf{v} \cdot \nabla\right) a=\quad \eta \nabla_{\perp}^{2} a+B_{0}^{\prime} \frac{\partial \psi}{\partial z^{\prime}},
\end{aligned}
$$

where $\nabla_{\perp}=\left(\partial_{x}, \partial_{y}, 0\right), \mathbf{v}=\nabla \times \psi \hat{\mathbf{z}}, \quad \mathbf{b}=\nabla \times a \hat{\mathbf{z}}, \omega=-\nabla_{\perp}^{2} \psi$, $j=-\nabla_{\perp}^{2} a$, and $\nabla_{\perp} \cdot \mathbf{v}=0$. This representation of fluctuations (but not explicitly the RMHD dynamical equations) has been adopted in closure models of "shear Alfvén wave" turbulence. ${ }^{13,14}$

Dissipation occurs via the action of both viscosity, $\nu$, and resistivity, $\eta$, but note that it involves $\nabla_{\perp}^{2}$ not the full $\nabla^{2}$. In these units $B_{0}^{\prime}$ is technically the (large-scale) Alfvén speed, $V_{A}^{\prime}$; for convenience, however, we often continue to refer to it as the mean field. Also, because of the rescaling of the $z$ coordinate, to $z^{\prime}$, lengthscale variations in this direction are necessarily large, i.e., $O(1)$. It is sometimes useful to emphasize this aspect by rewriting the final term in Eq. (1), for example, as

$$
\left(\frac{B_{0}^{\prime}}{\epsilon}\right)\left(\epsilon \frac{\partial}{\partial z^{\prime}}\right) j
$$

where a small parameter, $\epsilon$, appears explicitly (see Appendix A). The two bracketed terms then correspond directly to the actual (very strong) physical mean field $B_{0} \equiv B_{0}^{\prime} / \epsilon$, and the (very weak) gradient operator along it. ${ }^{3}$

It is worth emphasizing that RMHD is not a weak turbulence approximation. While it is true that the fluctuations are of small amplitude relative to $B_{0}$, and that the coupling between $z$-planes is only weakly nonlinear, the coupling within these planes is strongly nonlinear. This strong nonlinearity occurs as a consequence of the enforcement of the "hydrolike condition," as we now discuss.

The RMHD equations can be derived from the 3D MHD equations ${ }^{15}$ by assuming that (i) $v^{2}, b^{2} \ll B_{0}^{2}$, and (ii) that $\tau_{\mathrm{NL}} \lesssim \tau_{\mathrm{A}}$. We refer to the latter constraint, between the timescales, as the (global) hydrolike condition (see also Appendix B). More verbosely, it is the restriction that the nonlinear timescale for the fluctuations, $\tau_{\mathrm{NL}}=\lambda / \delta u$, is shorter than $\tau_{\mathrm{A}}=L_{\|} / B_{0}$, the time taken for an Alfvén wave(packet) to traverse a distance of order the typical lengthscale associated with parallel structure in the system, $L_{\|}$. Here, $\lambda$ is the energy-containing lengthscale for the velocity field and $\delta u$ is the rms velocity. (Often $\lambda$ is estimated using the correlation scale $\lambda_{c}$, which is a measurable quantity.) We refer to the simultaneous satisfaction of both assumptions (i) and (ii) above as satisfaction of the (global) RMHD condition.

It is also of interest to develop the analogous timescale condition in terms of Fourier modes. Fourier decomposing $\mathbf{v}(\mathbf{x})$ and $\mathbf{b}(\mathbf{x})$, one finds that there are two (nondissipative) timescales associated with wavevectors $\mathbf{k}$. These are,

$$
\begin{aligned}
& \tau_{\mathrm{A}}(\mathbf{k})=\frac{1}{\left|\mathbf{k} \cdot \mathbf{B}_{0}\right|}=\frac{1}{\left|k_{\|} B_{0}\right|}, \\
& \tau_{\mathrm{NL}}(\mathbf{k})=\frac{1}{k \widetilde{v}_{k}} \approx \frac{1}{k \widetilde{b}_{k}},
\end{aligned}
$$

respectively, referred to as the Alfvén (or wave) timescale and the (direction-averaged) modal nonlinear timescale. The quantity $\widetilde{v}_{k}^{2}$ is an approximation for the energy (per unit mass) associated with the Fourier components of $\mathbf{v}$ whose wavevectors have magnitude $k=|\mathbf{k}|$. (The near equality of $\widetilde{v}_{k}^{2}$ and $\widetilde{b}_{k}^{2}$ is a consequence of the Alfvén effect induced by the strong $B_{0},{ }^{16,17}$ but the definition based only on $\widetilde{v}_{k}$ should be retained in nonequipartition situations, as can occur due to boundary effects for instance. ${ }^{18}$ ) Note that two-dimensional (2D) modes have $k_{\|}=0$, by definition, and are nonpropagating with $\tau_{\mathrm{A}}(\mathbf{k}) \rightarrow \infty$. Thus, they always satisfy the hydrolike condition.

The ratio ${ }^{19}$ of these two timescales yields a parameter central to RMHD - and indeed MHD turbulence in general: $: 3,9,12,13,20-23$

$$
\epsilon_{\text {hydro }}(\mathbf{k})=\frac{\tau_{\mathrm{NL}}(\mathbf{k})}{\tau_{\mathrm{A}}(\mathbf{k})} .
$$

One can partition the Fourier modes into two sets on the basis of whether or not $0 \leqslant \epsilon_{\text {hydro }}(\mathbf{k}) \leqslant 1$ (referred to as the modal hydrolike condition). Those modes for which the inequality holds have a nonlinear time which is faster than the wave timescale and define the hydrolike modes. If, in addition to the hydrolike condition being satisfied, it is also true that the fluctuations are small amplitude in the sense that $\widetilde{v}_{k}^{2}, \widetilde{b}_{k}^{2} \ll B_{0}^{2}$, then we will call these $R M H D$ modes.

With a few further assumptions concerning the nature of the energy spectra, one can characterize regions of wavevector space that are distinguished according to the nature of 

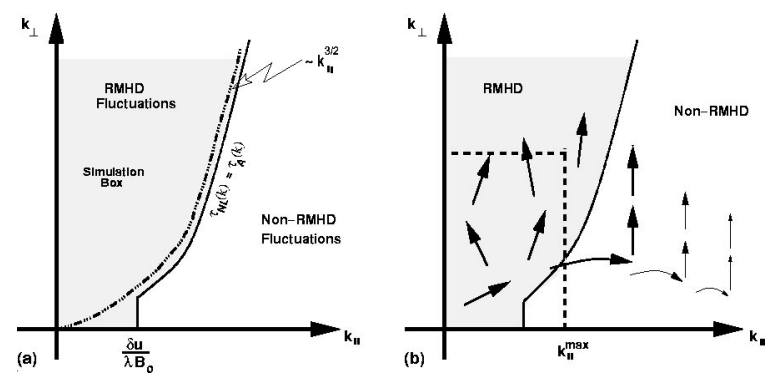

FIG. 1. (a) Cartoon sketch depicting the RMHD and non-RMHD regions, in Fourier space, and their boundary (solid curve) defined by the modal RMHD condition: $\tau_{\mathrm{NL}}(\mathbf{k})=\tau_{\mathrm{A}}(\mathbf{k})$, subject to the fluctuations being of small amplitude. The dash-dot curve represents the asymptotically valid form for the inertial range boundary, $k_{\perp} \sim\left(k_{\|} B_{0}\right)^{3 / 2}$. For illustration this has been inappropriately extended down to the origin. (b) Schematic indication of the direction of spectral transfer for RMHD and non-RMHD modes. The arrow thickness provides a rough guide to the strength of the transfer. Interactions inside the RMHD region are always resonant or nearly resonant, whereas outside this region resonant interactions can only occur at fixed $k_{\|}$(i.e., perpendicular transfer). The dashed box indicates a possible computational domain, with a maximum retained parallel wavenumber of $k_{\|}^{\max }$, and reveals that both kinds of modes can lie within such domains.

spectral transfer within them. Discussions along these lines have been given previously ${ }^{9,12,13,24-27}$ with a clear emphasis on understanding the nature of the highly anisotropic cascade $e^{9,28-31}$ that occurs in the directions perpendicular to a strong applied mean magnetic field. In the present paper the emphasis is on understanding the complementary issue of the nature of the parallel RMHD cascade.

Figure 1 illustrates some of the salient features of the MHD wavevector space in the presence of a strong $\mathbf{B}_{0}$. The shaded area indicates the region in which the RMHD condition is satisfied (we assume here that $\widetilde{v}_{k}, \widetilde{b}_{k} \ll B_{0}$ ). In the unshaded region, RMHD is not valid, and more complete MHD couplings are in principle required. One should note, however, that couplings involving RMHD modes occur in both regions, but in the latter region there are also couplings which do not involve RMHD modes (cf. Fig. 2). The boundary formed by the marginal RMHD condition separates the two regions. ${ }^{32}$
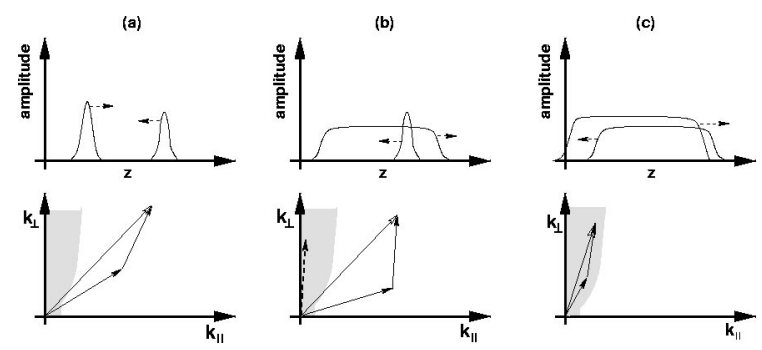

FIG. 2. Possible types of interactions between hydrolike and nonhydrolike wavepackets/modes. Shown are $\mathbf{x}$-space wavepacket interactions (top row) and corresponding $\mathbf{k}$-space wavevector triads (bottom row). In the bottom row, the shaded regions are indicative of the hydrolike regions and the driven mode (k) is depicted using an open arrow head. The mean field $\mathbf{B}_{0}$ is assumed to be at least somewhat strong. (a) Nonhydrolike with nonhydrolike. (b) Nonhydrolike with hydrolike. Note the nonlocal nature of the interaction in $\mathbf{k}$-space. The two near vertical arrows represent the same hydrolike mode, with the dashed one being its "natural" position and the solid one translated to form the triad. (c) Hydrolike with hydrolike.
To provide an estimate of the RMHD boundary in steady-state conditions, we suppose there is an energycontaining range of scales, characterized by a single (isotropic) length scale, $\lambda$. For $k \lambda<1$, the defining timescale inequality is $\lambda / \delta u<\left(k_{\|} B_{0}\right)^{-1}$. Consequently the RMHD region in the energy-containing range of scales lies within the region $k_{\|}<\lambda^{-1} \delta u / B_{0}$. For smaller (inertial range) scales, determination of the boundary requires a functional form for the spectrum. On assumption of a powerlaw Kolmogorov perpendicular energy spectrum $\sim k_{\perp}^{-5 / 3}$, and using $\tau_{\mathrm{NL}}(\mathbf{k})=(\lambda / \delta u)(1 / k \lambda)^{2 / 3}$, which matches the energycontaining range estimate at $k=1 / \lambda$, one estimates an inertial range RMHD region as $k_{\|}^{3}<\lambda^{-1}\left(\delta b / B_{0}\right)^{3}\left(k_{\|}^{2}+k_{\perp}^{2}\right)$. Figure 1(a) illustrates the RMHD region and its nominal boundary estimated using the marginal point of the above inequalities. As $B_{0}$ is increased the boundary draws in closer towards the $k_{\perp}$ axis, decreasing the size of the RMHD region.

Note that when $k_{\perp} \gg k_{\|}$the inertial range boundary asymptotically approaches the curve $k_{\perp} \sim\left(k_{\|} B_{0}\right)^{3 / 2}$. This is the boundary originally described by Higdon ${ }^{34}$ [below his Eq. (5)], and later described by Goldreich and Sridhar ${ }^{13}$ as the condition for a "critically balanced cascade." In the present estimate, the RMHD region is always somewhat larger than the estimate given by the asymptotic relation. The major difference is that in the energy-containing region $(k<1 / \lambda)$, the bandwidth of the RMHD region in $k_{\|}$does not approach zero, as it does when the asymptotic inertial range behavior is extrapolated-inappropriately-towards $k_{\perp}=0$.

Since the boundary is not rectilinear, a rectangular region of wavevector space, as would ordinarily be employed in a numerical simulation, will often include both RMHD and non-RMHD modes [Fig. 1(b)]. Exceptions to this could occur in a situation where the boundaries perpendicular to $\mathbf{B}_{0}$ are "open" (e.g., nonperiodic), and the implied wavelength of the fluctuations is larger than the simulation domain; see Appendix C.

From a numerical point of view, there is no particular problem in solving the RMHD equations using initial conditions which include non-RMHD fluctuations, as would be the case for the situation sketched in Fig. 1(b), for example. Presumably, at later times the solutions to the equations will still include contributions from non-RMHD fluctuations. Questions then arise as to the consistency of the RMHD equations, including whether they adequately capture the physics of non-RMHD fluctuations along with that of the RMHD ones.

The question we wish to address herein is whether spectral transfer is eventually expected to invalidate the assumptions of RMHD. Two initial situations, in particular, are of interest. First, if a simulation is started with excitations confined within the RMHD wavevector region, will they always remain so confined? If not, how dynamically significant is the generation of non-RMHD modes? Second, if the initial data includes both RMHD and non-RMHD fluctuations, will a region of non-RMHD fluctuations grow in importance as time increases? Clearly, these are issues that depend upon the rate and nature of parallel spectral transfer from the RMHD region into the non-RMHD region. 


\section{SPECTRAL TRANSFER: PERPENDICULAR VERSUS PARALLEL}

The resonance condition for spectral transfer in (full) incompressible MHD with a $\mathbf{B}_{0}$ has been discussed extensively ${ }^{9-13,21,24-26,28-31,35-40}$ and cited as a partial justification for the validity of the RMHD approximation. ${ }^{3}$ The mathematical context emerges by imposing frequency matching (i.e., resonance) in addition to the mandatory wavevector matching of the triad (aka three "wave") interactions arising from the MHD quadratic nonlinearities. This provides conditions, akin to selection rules, for rapid nonlinear couplings that drive the cascade.

It is convenient to discuss this in terms of an Elsässer representation in which right-traveling fluctuations are $\mathbf{z}^{-}$ $=\mathbf{v}-\mathbf{b}$, say, and left-traveling fluctuations are $\mathbf{z}^{+}=\mathbf{v}+\mathbf{b}$, relative to a rightwards directed mean magnetic field $\mathbf{B}_{0}$, which is assumed to be at least as energetic as the fluctuations. Two features make resonance difficult to achieve. First, and rather remarkably, Alfvén waves propagating in the same direction do not interact nonlinearly at all, even for finite amplitudes. Second, oppositely propagating wavepackets can only interact for a time of the order of their crossing time $\tau_{\text {int }}=\lambda_{\|} /\left(2 V_{A}\right)$, where $\lambda_{\|}$is the larger of the parallel extents of the two propagating packets (Fig. 2).

For simplicity, assume that the left and right-traveling packets have dominant characteristic wavevectors, respectively $\mathbf{p}$ and $\mathbf{q}$. Thus,

$$
\begin{aligned}
& \mathbf{z}^{+}(\mathbf{x}, t) \sim \mathbf{A}_{p}^{+}(t) \mathrm{e}^{i\left(\mathbf{p} \cdot \mathbf{x}-\omega_{p} t\right)}, \\
& \mathbf{z}^{-}(\mathbf{x}, t) \sim \mathbf{A}_{q}^{-}(t) \mathrm{e}^{i\left(\mathbf{q} \cdot \mathbf{x}-\omega_{q} t\right)},
\end{aligned}
$$

where the sense of propagation is encoded in the choice of the wave frequencies: $\omega_{p}=-p_{\|} B_{0}$ and $\omega_{q}=+q_{\|} B_{0}$. Note that the amplitudes $\mathbf{A}^{ \pm}$are in general time-dependent, although this dependence may turn out to be "slow." We define the cross helicity as $H_{c}=\left(\mathbf{z}^{+} \cdot \mathbf{z}^{+}-\mathbf{z}^{-} \cdot \mathbf{z}^{-}\right) / 4$, which is positive for left-traveling modes and negative for righttraveling ones. ${ }^{41}$

The two packets can interact to drive left and righttraveling packets with (the same) dominant wavevector $\mathbf{k}$ $=\mathbf{p}+\mathbf{q}$ and amplitudes $\mathbf{A}_{k}^{ \pm}$. Substituting into the Elsässer form of the MHD equations $\left(\partial \mathbf{z}^{ \pm} / \partial t \sim-\mathbf{z}^{\mp} \cdot \nabla \mathbf{z}^{ \pm} \pm \mathbf{B}_{0}\right.$ $\cdot \nabla \mathbf{z}^{ \pm}$), one obtains for the right-traveling packet,

$$
\begin{aligned}
& \mathrm{e}^{i\left(\mathbf{k} \cdot \mathbf{x}-k_{\|} B_{0} t\right)} \frac{\partial \mathbf{A}_{k}^{-}}{\partial t} \sim \mathbf{q} \cdot \mathbf{A}_{p}^{+} \mathbf{A}_{q}^{-} \mathrm{e}^{i(\mathbf{p}+\mathbf{q}) \cdot \mathbf{x}^{-i\left(\omega_{p}+\omega_{q}\right) t}} \\
& \Rightarrow \frac{\partial \mathbf{A}_{k}^{-}}{\partial t} \sim \mathbf{q} \cdot \mathbf{A}_{p}^{+} \mathbf{A}_{q}^{-} \mathrm{e}^{i 2 p_{\|} B_{0} t},
\end{aligned}
$$

upon substitution of the (parallel) wavevector matching condition $k_{\|}=p_{\|}+q_{\|}$. A similar equation holds for $\partial \mathbf{A}_{k}^{+} / \partial t$. Exact resonance can only occur if the argument to the exponential is zero, which implies that $p_{\|}=0$. Thus, the mode which was assumed to be left-traveling, must in fact have a zero parallel wavenumber and is therefore a nonpropagating mode (with, however, the same sign of cross helicity as a left- traveling mode), as was first pointed out by Shebalin et al. ${ }^{31}$ The nonpropagating modes are also known as the 2D turbulence modes. 42

Similarly, resonant driving of $A_{k}^{+}$requires that $q_{\|}=0$. Thus, a 2D mode and a propagating mode (of the opposite sign of cross helicity) interact to drive another mode with the same propagation properties as the driving mode. In particular, the driven and driving modes have the same parallel wavenumber, so that driving occurs at constant $k_{\|}$. At this order, then, there is no parallel spectral transfer and the energy cascade is purely perpendicular. ${ }^{25,28-31}$

The above conditions for exact resonance can be relaxed, by allowing the argument of the exponential term to be small rather than zero. This yields the conditions for quasi-resonance. Here, small means that averaging the righthand side of Eq. (10) over the Alfvén time associated with the driving wave does not give a near-zero result. Thus, if $T_{q}=2 \pi /\left(q_{\|} B_{0}\right)$, and $\mathbf{A}_{p}^{+}$and $\mathbf{A}_{q}^{-}$do not vary appreciably over this timescale, then one requires

$$
\frac{1}{T_{q}} \int_{0}^{T_{q}} \mathrm{e}^{i 2 p_{\|} B_{0} t} \mathrm{~d} t \approx 1 \Rightarrow \frac{2 p_{\|}}{q_{\|}} \ll 1 .
$$

Hence, provided that one of the wavepackets is very broad compared to the other, quasi-resonance will occur when they collide [Fig. 2(b)].

There is an additional restriction, if the notion of "quasiresonance" is to mean that nonlinear interactions can proceed unabated by wave propagation effects. That is, the beat frequency associated with the inexactness of the resonance must be low enough that the nonlinearity is not averaged to zero. Accordingly we require that

$$
\left|\omega_{p}+\omega_{q}-\omega_{k}\right|<\frac{1}{\tau_{\mathrm{NL}}(\mathbf{k})} .
$$

The above understanding of quasi-resonance leads to an improved picture for the interaction of MHD wavepackets. Since any system with a $B_{0}$ can always support a hydrolike region, ${ }^{43}$ it is useful to treat each wavepacket as being either a hydrolike $(H)$ one or a nonhydrolike $(N)$ one. There will then be three distinct classes of interaction: $N-N, N-H$, and $H-H$ (Fig. 2). These classes are now discussed in more detail.

\section{A. Nonhydrolike-nonhydrolike interactions}

This corresponds to the interaction of (counterpropagating) high-frequency waves, essentially via the wellknown Iroshnikov-Kraichnan picture, ${ }^{44-46}$ with $\tau_{\text {int }} \ll \tau_{\mathrm{NL}}$. Since both interacting modes have large parallel wavenumbers, resonance is not possible [Fig. 2(a)]. If the interactions were coherent it would take $n=\tau_{\mathrm{NL}} / \tau_{\text {int }}$ encounters to amass the equivalent of a single collision of duration $\tau_{\mathrm{NL}}$. However, as they are incoherent, $n^{2}$ encounters are required, giving a spectral transfer time of $\tau_{\mathrm{s}}=n^{2} \tau_{\text {int }}=\tau_{\mathrm{NL}}^{2} / \tau_{\text {int }} \gg \tau_{\mathrm{NL}}$, so that relative to hydrodynamics the cascade process is significantly slowed.

The wavenumber scaling of the inertial range associated with this interaction class is a question of some interest. Recall that the Kolmogorov dimensional analysis approach to 
inertial range cascades-originally written in terms of the nonlinear timescale $\tau_{\mathrm{NL}}(\mathbf{k})$ - can be reformulated in terms of the triple correlation timescale $\tau_{3}(\mathbf{k})$, as was first done by Kraichnan. ${ }^{45,46}$ Under the assumption of isotropy this yields

$$
\epsilon \approx \tau_{3}(k) k^{4} E^{2}(k),
$$

where $\epsilon$ is the spectral energy flux, $k=|\mathbf{k}|$, and $E(k)$ is the omni-directional energy spectrum. The IroshnikovKraichnan scaling follows by taking $\tau_{3}=1 / k B_{0}$ and assuming small cross helicity. ${ }^{44,45}$ However, for the present class of interactions isotropy is clearly a poor assumption. Interestingly though, using the knowledge that the cascade is primarily perpendicular one can employ an anisotropic variant of Eq. (13) to obtain a scaling which agrees with that of more formal analyses ${ }^{25,26}$ (see also Refs. 11, 18, 35, and 37), as we now show. When $B_{0}$ is strong and the parallel cascade is suppressed, the energy transfer rate $\epsilon$ is almost entirely due to couplings to higher perpendicular wavenumber, and the nonlinear timescale should depend primarily upon $k_{\perp}$. On the other hand wave propagation effects are associated with $\tau_{3}(\mathbf{k})=1 /\left|k_{\|} B_{0}\right|$, the (anisotropic) Alfvén timescale. Ignoring the dependence of the energy spectrum on $k_{\|}$, Eq. (13) is rearranged to find

$$
E\left(k_{\perp}\right)=k_{\perp}^{-2} \sqrt{\epsilon_{\perp}\left(k_{\|}\right) k_{\|} B_{0}} \sim k_{\perp}^{-2},
$$

where $\epsilon_{\perp}\left(k_{\|}\right)$is the (perpendicular) energy flux at that value of $k_{\|}$. This scaling with $k_{\perp}$ has been observed in direct numerical simulations of RMHD under certain boundary conditions which limit the efficiency of the perpendicular cascade. ${ }^{18}$ Note that the dependence on $k_{\|}$is as a parameter not a variable. The functional form of the parallel energy spectrum is undetermined in this approach. ${ }^{25}$

\section{B. Nonhydrolike-hydrolike interactions}

Interactions of this type are essentially the same as those first identified by Shebalin et al., ${ }^{31}$ but with allowance made for quasi-resonance rather than exact resonance. As discussed in connection with Eq. (11) above, the quasi-resonant spectral transfer occurs at quasi-constant $k_{\|}$[Fig. 2(b)]. That is, the turbulent cascade is an approximately perpendicular one.

Clearly, the efficiency of parallel spectral transfer is weak for these interactions. Indeed, there are good grounds for expecting the parallel spectrum to have an exponential falloff, a suggestion first made by Montgomery. ${ }^{47}$ Section IV A contains more discussion on this matter.

Note that these interactions are strongly nonlocal in k-space, with resonant driving of the high-frequency $(N)$ modes requiring the presence of "catalytic" hydrolike modes. The nonlocality is due to the possibly substantial difference in parallel wavenumber [see Fig. 2(b)] for $N$ and $H$ modes. Moreover, the strength of the interaction depends on the partitioning of energy between the $H$ and $N$ modes. ${ }^{9,12,25}$

Under the condition that the quasi-two-dimensional part of the spectrum (the $H$ modes) is sufficiently strong, it seems likely that the perpendicular spectrum of the $N$ modes will be determined by the $\mathrm{N}-\mathrm{H}$ interactions, and that this would lead to an expected $k_{\perp}^{-5 / 3}$ spectrum in the $N$ range of $k_{\|}$. The weak turbulence contribution of $k_{\perp}^{-2}$ would be masked. On the other hand, the whole relevance of the expected direct transfer inertial range for the $N-N$ and $N-H$ interactions may be called into question if, at a given $k_{\|}$, the spectrum needs to climb "up" at large $k_{\perp}$ to meet the hydrolike boundary (cf. Fig. 4).

\section{Hydrolike-hydrolike interactions}

As a consequence of the inherently low frequencies associated with hydrolike modes, the interaction time for two $H$ modes is long, and will typically be associated with driving of another $H$ mode. In this sense their self-interactions are always nearly resonant, sometimes referred to as "trivially resonant." 48 Thus, the hydrolike modes constitute a resonantly broadened analog of the strictly 2D modes. (Clearly, the 2D-2D and 2D-H interactions are subsets of this interaction class.) These are also sometime called "zero modes" and their couplings amongst one another, zero frequency turbulence. Typically, it will not be particularly useful to think of these modes as being wave-like.

For excitations well within the hydrolike region, parallel and perpendicular spectral transfer are not essentially different, since wave effects are secondary there. Consequently, the spectral transfer is approximately isotropic, although its region of applicability is not, and indeed emphatically not when $B_{0}$ is strong [Fig. 2(c)]. Transfer in the perpendicular direction proceeds unimpeded until the (perpendicular) dissipation scale, $k_{\text {diss }}$, is reached. However, as the hydrolike region is narrow in the parallel direction, transfer in this direction soon encounters the hydrolike boundary region where $\tau_{\mathrm{NL}} \approx \tau_{\mathrm{A}}$.

Once the excitation is transferred across this boundary, into an $N$ mode, it is no longer in the always (nearly) resonant region and its subsequent dynamics are of the $N-H$ or $N-N$ kind, discussed above. ${ }^{49}$ A sharp "energy cliff" will thus develop around the hydrolike boundary zone ${ }^{13}$ (see Sec. IV).

The shape of the parallel spectrum inside the hydrolike region may be rather difficult to determine and problem dependent. When $B_{0}$ is large, there are relatively few $k_{\|} \mathrm{s}$ in the hydrolike region-especially at low $k_{\perp}$-so that there is little scope for the development of an inertial range or other scaling range in this direction. The simulations discussed below suggest that treating the parallel spectrum as approximately flat in this region is a reasonable approach (cf. Refs. 13, 25, and 26).

As a final point in this section, we note that since the energy of the (strictly) 2D modes is continually cascaded towards the dissipative sink at high $\mathbf{k}_{\perp}$, there is a need to replenish this energy if this component is to survive. It is straightforward to show that exact resonant driving of 2D modes using non-2D modes is not possible. However, nearly resonant replenishment of the $2 \mathrm{D}$ modes can occur, via the interaction of two hydrolike modes, $\mathbf{p}$ and $\mathbf{q}$, with $p_{\|}$ $=-q_{\|}$. In the absence of external forcing of the $2 \mathrm{D}$ modes, we suggest that this is the primary mechanism for replenishment of their energy. 

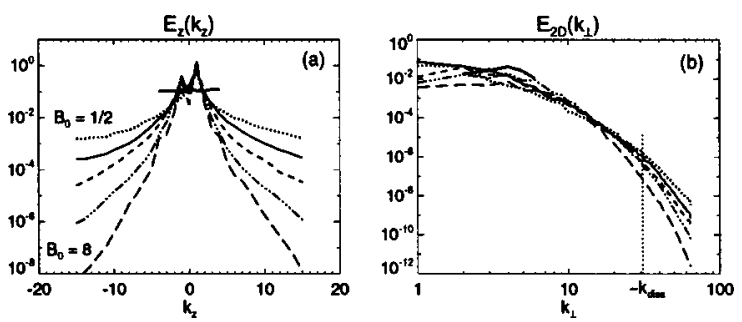

FIG. 3. Steady-state energy spectra from $128^{2} \times 32$ simulations with $B_{0}^{\prime}$ $=1 / 2,1,2,4,8$. (a) Parallel spectra. (b) Perpendicular spectra for the 2D $\left(k_{z}^{\prime}\right.$ $=0)$ modes. The perpendicular dissipation wavenumber is indicated. Curves are ordered with $B_{0}^{\prime}$. The "standard" case of $B_{0}^{\prime}=1$ is depicted using a solid curve. In each plot, the thick roughly horizontal line is the initial condition.

\section{SIMULATIONS}

Numerous spectral method (Fourier collocation) simulations of the RMHD equations were performed with values of $B_{0}^{\prime}$ between $1 / 2$ and 8 (cf. Appendix A). The resolutions employed included $128^{2} \times 32$, and $64^{2} \times N_{z}$ with $N_{z}=16,32$, and 64 . Time advancement was via a second-order RungeKutta algorithm. To achieve steady-state conditions the RMHD equations were augmented with forcing terms and run for some 40-50 box-crossing times. The forcing terms were originally designed for a coronal heating model,,${ }^{50}$ and consist of directly driving a single Alfvén wave with wavevector $\mathbf{k}=(1,1,1)$, and allowing all modes to undergo reflection at a specified rate. All the runs used initial conditions (ICs) where the excited modes were restricted to have $2 \leqslant k_{\perp} \leqslant 6$ and $-K_{z} \leqslant k_{z}^{\prime} \leqslant K_{z}$ with approximately equal amounts of energy in each $k_{z}^{\prime}$ plane. Usually we set $K_{z}=4$. The fluctuations were initialized in Fourier space using Gaussian random phases, with the amplitudes of $\mathbf{v}(\mathbf{k})$ and $\mathbf{b}(\mathbf{k}) \sim 1 / \sqrt{1+\left(k_{\perp} / 5\right)^{8 / 3}}$. The initial energy in the fluctuations was unity, equipartitioned between the velocity and magnetic fields, with normalized cross helicities $\sim 0.02$. All runs where performed with $\nu=\eta=1 / 200$, corresponding to initial large-scale Reynolds numbers of 200.

\section{A. Strength and nature of the cascades}

Figure 3(a) shows the parallel energy spectrum, $E_{z}\left(k_{z}^{\prime}\right)$, for five $128^{2} \times 32$ runs, each employing the same initial state but with distinct values of $B_{0}^{\prime}$. The parallel spectrum is defined as

$$
E_{z}\left(k_{z}^{\prime}\right)=\sum_{k_{x}, k_{y}} E\left(k_{x}, k_{y}, k_{z}^{\prime}\right),
$$

where $E(\mathbf{k})$ is the full (kinetic plus magnetic) modal energy spectrum of the fluctuations. Clearly there is significant falloff with increasing $\left|k_{z}^{\prime}\right|$ for all values of $B_{0}^{\prime}$, and the stronger $B_{0}^{\prime}$ the more dramatic the falloff. Although the range of $k_{z}^{\prime}$ 's employed is not large, fits to an exponential dependence on $\left|k_{z}^{\prime}\right|$ for $\left|k_{z}^{\prime}\right| \gtrsim 7$ are reasonably convincing, particularly for larger $B_{0}^{\prime}$. Note that the (steady-state) Reynolds numbers for these runs have deliberately been kept rather low $\left(R_{e} \approx R_{m}\right.$ $\approx 200-300$ ) to ensure that there is no doubt regarding the resolution of the perpendicular dissipation scale.
Similar results are obtained for various other resolutions and initial conditions we have investigated. In particular, for a given runset (i.e., same IC, different $B_{0}^{\prime}$ values) the parallel spectra are always ordered with $B_{0}^{\prime}$ in the same way as they are in Fig. 3(a).

Also shown [Fig. 3(b)], for the same set of $B_{0}^{\prime}$ values, are the steady perpendicular spectra for the $k_{z}^{\prime}=0$ (2D) modes. We define $E_{2 \mathrm{D}}\left(k_{\perp}\right) \equiv E_{\text {axi }}\left(k_{z}^{\prime}=0, k_{\perp}\right)$, where the axisymmetric energy spectrum (at given $k_{\perp}$ and $k_{z}^{\prime}$ ) is

$$
E_{\text {axi }}\left(k_{z}^{\prime}, k_{\perp}\right)=\sum_{k_{x}, k_{y} \text { with }\left|k_{\perp}-\sqrt{k_{x}^{2}+k_{y}^{2}}\right|<1 / 2} E\left(k_{x}, k_{y}, k_{z}^{\prime}\right) .
$$

The $E_{2 \mathrm{D}}$ spectra are qualitatively similar for all the $B_{0}^{\prime}$, and there is even a short region around $k_{\perp} \sim 8-20$ where they are, very roughly, equal. This relatively weak dependence upon $B_{0}^{\prime}$ is in marked contrast to the situation for the parallel spectra. The Reynolds numbers are too low for genuine (perpendicular) inertial ranges to develop.

We now analyze the possibility that the parallel spectrum is exponential in character. Consider first the situation in full 3D MHD (cf. Sec. III). From the (parallel) wavevector matching condition, $k_{\|}=p_{\|}+q_{\|}$, it is clear that parallel spectral transfer can only occur if both $p_{\|}, q_{\|} \neq 0$. The exponential factor in Eq. (10) is then always oscillatory, with angular frequency $\omega_{\text {chop }}=2 p_{\|} B_{0}$, and exact resonance is unachievable. As $B_{0}$ and/or $p_{\|}$are increased in magnitude, the "chopping" effect of this term becomes increasingly pronounced since

$$
\Delta \mathbf{A}_{k}^{-} \approx \int_{t_{1}}^{t_{2}} \mathbf{q} \cdot \mathbf{A}_{p}^{+}(t) \mathbf{A}_{q}^{-}(t) \mathrm{e}^{i 2 p_{\|} B_{0} t} \mathrm{~d} t
$$

is more effectively averaged towards zero.

For a given simulation $B_{0}$ is fixed. Over the (short) interval $t_{2}-t_{1}$ many different values of $p_{\|}$will contribute to the total ${ }^{51} \Delta \mathbf{A}_{k}^{-}$. For parallel transfer, the most effective contributions will typically come from the smaller $p_{\|}$'s (usually associated with RMHD modes). Recall, that in a periodic system the wavevectors are discretely spaced so that there is a minimum difference between distinct wavevectors, i.e., $\left|\mathbf{k}_{1}-\mathbf{k}_{2}\right| \geqslant \Delta k_{\min }$. It follows that parallel cascade must be associated with changes in parallel wavenumber quantized in units of $\Delta k_{\text {min }}$. Similarly $\omega_{\text {chop }}=2 p_{\|} B_{0} \equiv 2\left(k_{\|}\right.$ $\left.-q_{\|}\right) B_{0}$ is quantized in units of $2 \Delta k_{\min } B_{0}$. Thus, the smallest non-zero $p_{\|}$has magnitude $\Delta k_{\min }$ and implies $k_{\|}=q_{\|}$ $\pm \Delta k_{\min }$, and an associated parallel cascade. The efficacy of this cascade will clearly be weakened for larger values of $p_{\|}$.

However, as noted by Kinney and McWilliams, ${ }^{27}$ it is not a cascade in the traditional sense since Eq. (17) depends upon the (mode-independent) amplitude $B_{0}$. Consequently, it is not clear that power-law scalings in $k_{\|}$will result. Indeed, since the perpendicular cascade is, at leading order, both resonant and $B_{0}$-independent, it is likely that at a given $k_{\|}$ most of the energy will be transferred to high $k_{\perp}$, with only a small amount trickling through to still higher $k_{\|}$[cf. Fig. 1(b)]. This "survivalist" interpretation of the parallel transfer also suggests that the parallel spectrum will be exponential. 

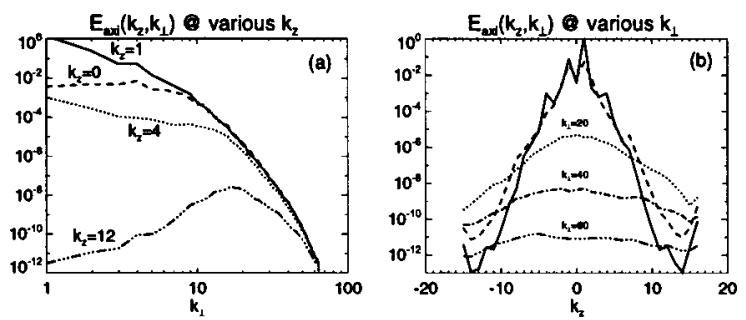

FIG. 4. Several cross-sections through the spectrum $E_{\text {axi }}\left(k_{z}^{\prime}, k_{\perp}\right)$ for the $128^{2} \times 32$ run with $B_{0}=8$. (a) $E_{\text {axi }}\left(k_{z}^{\prime}, k_{\perp}\right)$ at indicated $k_{z}^{\prime}$. Note that for $k_{z}^{\prime}=12$, the perpendicular spectrum actually shows a large increase between $k_{\perp}=1$ and $k_{\perp} \approx 20$. (b) $E_{\text {axi }}\left(k_{z}^{\prime}, k_{\perp}\right)$ at $k_{\perp}=1$ (solid), 4 (dash), and several indicated values. Regions which are approximately flat in $k_{z}^{\prime}$ increase in width as $k_{\perp}$ increases. The dissipation wavenumber in the perpendicular direction is $\approx 32$.

An exponential form for the parallel spectrum of (full) 3D MHD turbulence with a strong $B_{0}$ was first suggested by Montgomery. ${ }^{47}$ More recently, Kinney and McWilliams ${ }^{9}$ made a similar suggestion within the context of RMHD turbulence, and also provided evidence for it from freely decaying RMHD simulations. The numerical support presented herein appears to be the first for the case of forced (steady) RMHD turbulence.

Plots of the steady axisymmetric energy spectrum, defined by Eq. (16), are also illuminating. Figure 4 shows several cross-sections of this quantity from the $128^{2} \times 32$ run with $B_{0}=8$. The lefthand panel displays cross-sections at four values of $k_{z}^{\prime}$. The striking similarity of the curves at high $k_{\perp}$ suggests that within the RMHD regions parallel transfer is efficient, producing parallel spectra that are approximately flat. This is corroborated in Fig. 4(b). Note that at low $k_{\perp}$, the falloff with $k_{z}^{\prime}$ is so sharp that at $k_{z}^{\prime}=12$ (for example) the perpendicular spectrum actually has to increase by a factor of $\approx 10^{4}$ between $k_{\perp}=1$ and $\approx 20$ (possibly via "back-fill" from larger $k_{\perp}$ ), in order to attain the correct spectral level near the $\tau_{\mathrm{NL}}(\mathbf{k}) \approx \tau_{\mathrm{A}}(\mathbf{k})$ boundary.

Figure 4(b) displays cross-sections in the perpendicular direction, at five values of $k_{\perp}$. The efficiency of parallel transfer within the RMHD region, especially at larger $k_{\perp}$, is apparent from the flatness of the cross-sections there. Note, moreover, that the $k_{\perp}=1$ and $k_{\perp}=4$ curves in this panel have, more or less, the same $k_{z}$ boundary, especially for $\left|k_{z}^{\prime}\right| \leqslant 5$. This provides support for our contention that the shape of the RMHD boundary at low $k_{\perp}$ is determined by the large-scale eddy turnover time (see the discussion regarding Fig. 1 in Sec. II).

In summary, the simulation results support the assertion that RMHD is a self-consistent model. In particular, the transfer of energy from RMHD modes to non-RMHD ones is found to be a weak process associated with steep, possibly exponential, perpendicular spectra.

\section{B. Resolution issues}

The results of the previous subsection show that parallel cascade in RMHD is indeed self-limiting, due to the weakness of spectral transfer in this direction, while perpendicular transfer continues to drain away available energy. There is,
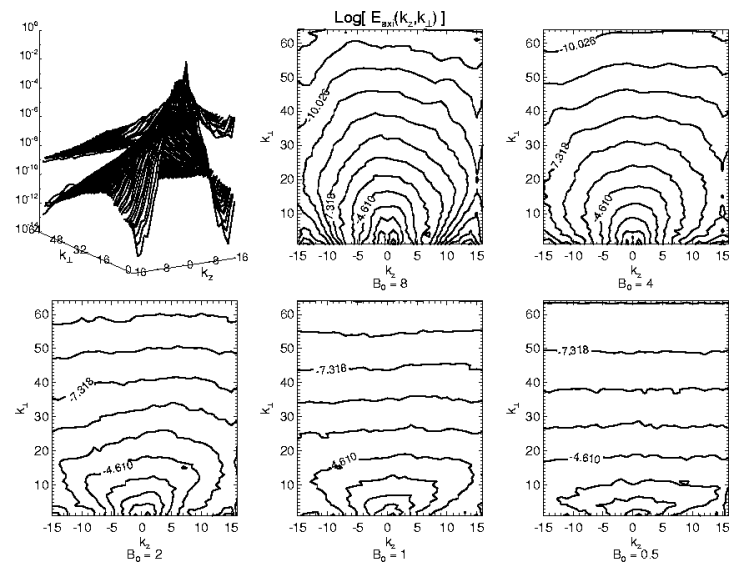

FIG. 5. Steady energy spectra, $E_{\text {axi }}\left(k_{z}^{\prime}, k_{\perp}\right)$, from fixed resolution $128^{2}$ $\times 32$ simulations for various $B_{0}^{\prime}$. Top left: Overlaid surface plots for $B_{0}^{\prime}$ $=1$ and 8. Remaining panels: Contour plots of $\log E_{\mathrm{axi}}\left(k_{z}^{\prime}, k_{\perp}\right)$ for the indicated values of $B_{0}^{\prime}$, using the same fixed contour levels in each plot. The putative size of the RMHD region (as indicated by the area where the contours are roughly parallel to the $k_{z}^{\prime}$-axis) is seen to increase as $B_{0}^{\prime}$ is decreased. However, for this parallel resolution $\left(k_{z}^{\max }=16\right)$, probably only the $B_{0}^{\prime}=8$ run is adequately resolved.

however, still the question of how much resolution is required in the parallel direction in order to adequately resolve both the parallel and the perpendicular structure. In effect, this is the question of how to choose $B_{0}^{\prime}$ and $k_{z}^{\max }$ (defined to be the maximum $k_{z}^{\prime}$ retained in the simulation), in tandem, in such a way that the spectra are well-resolved in all directions. (The usual requirement that the minimum perpendicular scale resolved is smaller than the dissipation scale is also needed.)

Since the above arguments and simulation results indicate that the evolution of non-RMHD modes is handled adequately by the RMHD equations, ideally the simulation domain should be chosen large enough to ensure that the RMHD region is a proper subset of it. This is perhaps easiest to see in Fourier space [e.g., Fig. 1(b)]. If, instead, the simulation boundary $k_{z}^{\max }=\max \left\{\left|k_{z}^{\prime}\right|\right\}$ crosses into the true RMHD region at higher values of $k_{\perp}$, as in Fig. 1(b), then the system is likely to be improperly resolved. In particular, energy which should have been transferred within a widening RMHD region may be constrained to transfer through an artificially narrow RMHD region, leading to elevated spectral levels and changes of spectral slopes there. Although global quantities such as the energy decay rates could conceivably still be correct in such simulations, the spectral distribution of energy, etc. clearly will not be.

We examine numerically the influence of parallel resolution using two complementary studies. In the first, $B_{0}^{\prime}$ is varied with the resolution held fixed, while in the second $k_{z}^{\max }$ is varied with $B_{0}^{\prime}$ fixed. Figure 5 displays plots from the first such study. Shown are contours and surfaces of the steady-state axisymmetric energy spectra, for five $128^{2} \times 32$ runs with $B_{0}^{\prime}=1 / 2,1,2,4$, and 8 . The apparent size of the RMHD region appears to increase as $B_{0}^{\prime}$ is decreased. This shows that there is insufficient parallel resolution since the boundary of the RMHD region is not evident. Indeed, probably only the $B_{0}^{\prime}=8$ case is properly resolved here. The pres- 

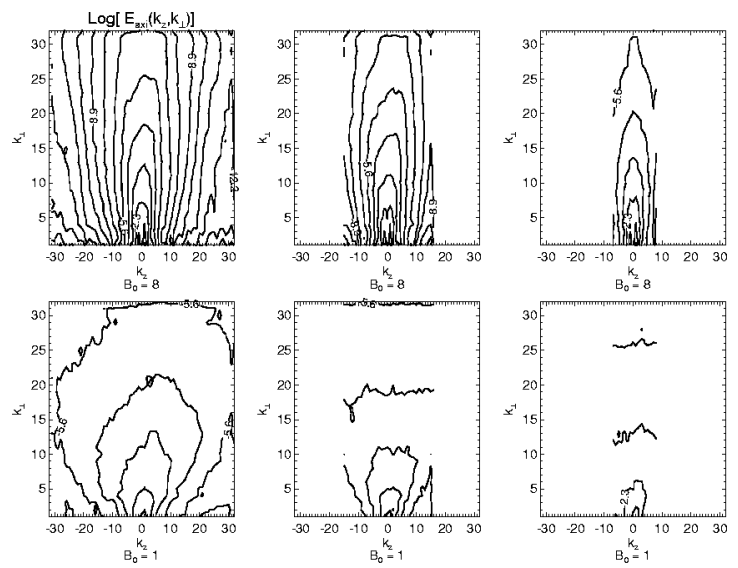

FIG. 6. Contour plots of the (log of) the energy spectra $E_{\text {axi }}\left(k_{z}^{\prime}, k_{\perp}\right)$ showing improving resolution of parallel transfer as the maximum retained $k_{z}^{\prime}$ is increased. Simulations are at $64^{2} \times\{64,32,16\}$. Top row: $B_{0}^{\prime}=8$ case. Bottom row: $B_{0}^{\prime}=1$ case. All plots use the same set of (equi-spaced) contour levels.

ence of approximately horizontal contours which span the entire $k_{z}^{\prime}$ domain is diagnostic of under-resolution in this direction. Note also that the energy is piling up in $k_{\perp}$ as $B_{0}^{\prime}$ is decreased (consider, for example, the " -7.318 " contours).

Results from the second study are shown in Figs. 6 and 7. The former displays contour plots of the (steady) axisymmetric energy spectra. Plots are shown for two different values of $B_{0}^{\prime}$, each at three parallel resolutions. For the $B_{0}^{\prime}=8$ cases (top row) retaining 32 or 64 parallel wavenumbers gives adequate resolution, while retaining 16 is probably not quite adequate. As can be seen from inspection of the location of the " -5.6 " contours, for example, there is a definite accumulation of energy well above the level seen in the two more highly resolved cases. For the $B_{0}^{\prime}=1$ cases, only the $64^{2} \times 64$ run shows signs of an encircling non-RMHD region, and even it may only be marginally resolved. Empirically, then, for these simulations it seems that acceptable parallel resolution can be ensured if $k_{z}^{\max } B_{0}^{\prime} \approx 100$. On the other hand, if contour plots of $E_{\text {axi }}\left(k_{z}^{\prime}, k_{\perp}\right)$ include some contours which are "flat" across all $k_{z}^{\prime}$, then the parallel resolution is unlikely to be adequate.

More generally, one can obtain a lower bound on the product $k_{z}^{\max } B_{0}^{\prime}$, which should be comfortably exceeded to ensure adequate parallel resolution. The bound is obtained by evaluating $\tau_{\mathrm{NL}}(\mathbf{k})=\tau_{\mathrm{A}}(\mathbf{k})$ at a $k_{\perp}$ equal to the Kolmogorov dissipation wavenumber. As usual $k_{\mathrm{diss}}=\left(\epsilon / \nu^{3}\right)^{1 / 4}$, where $\epsilon$ is the mean energy dissipation rate, assumed equal to the
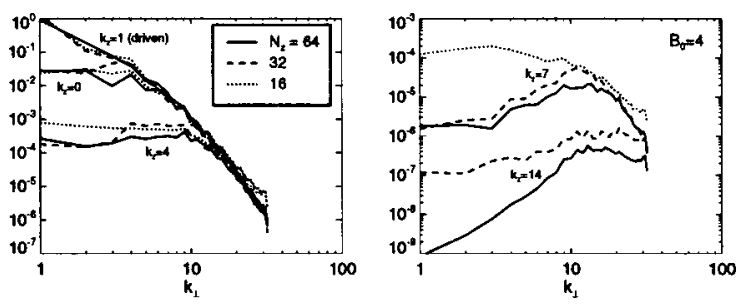

FIG. 7. Cross-sections of $E_{\text {axi }}\left(k_{z}^{\prime}, k_{\perp}\right)$ at various $k_{z}^{\prime}$ from $B_{0}^{\prime}=4$ runs at three different parallel resolutions: $64^{2} \times N_{z}$. mean forcing rate at steady state, approximated as $(\delta u)^{3} / \lambda$. Equivalently, one can use the inner timescale $\tau_{\text {diss }}=\sqrt{\nu / \epsilon}$ in place of $\tau_{\mathrm{NL}}(\mathbf{k})$. Both approaches yield

$$
k_{z}^{\max } B_{0}^{\prime}=\frac{1}{\tau_{\mathrm{NL}}} R_{e}^{1 / 2},
$$

where, as before, $\tau_{\mathrm{NL}}=\lambda / \delta u$ is the global nonlinear timescale. Although this formula is likely to underestimate the actual product required-since it makes no allowance for an encircling non-RMHD region-it does reveal the scaling with Reynolds number. For example, the simulations associated with Fig. 6 have $R_{e} \approx 250$ and eddy turnover times of order one, giving $k_{z}^{\max } B_{0}^{\prime} \approx 16$, which is well below the empirical value of 100. Equation (18) can also be rewritten to give a useful expression for the ratio of the required parallel and perpendicular resolutions:

$$
\frac{k_{z}^{\max }}{k_{\mathrm{diss}}}=\frac{\delta b}{B_{0}^{\prime}}\left(\frac{1}{k_{\mathrm{diss}} \lambda}\right)^{1 / 3},
$$

where $\delta b \approx \delta u$ is assumed.

More information on the character of the errors in the energy spectra arising from lack of parallel resolution is revealed in Fig. 7. Shown are perpendicular cross-sections of $E_{\text {axi }}\left(k_{z}^{\prime}, k_{\perp}\right)$ taken from three $B_{0}^{\prime}=4$ runs with differing parallel resolutions. Run parameters are identical except for the value of $N_{z}$. The left-hand panel indicates that all three resolutions are acceptable at low enough $k_{z}^{\prime}$. Indeed, for $k_{z}^{\prime}=0$ and $k_{z}^{\prime}=1$, the traces from the different resolution runs are usually overlain. The same is true for $k_{z}^{\prime}=4$ at sufficiently large $k_{\perp}$. At smaller $k_{\perp}$, the lowest resolution run is out by a factor of $\sim 3$, suggesting that the resolution is inadequate. This is confirmed by examining cross-sections for two other representative values of $k_{z}^{\prime}$ (right-hand panel). For the $k_{z}^{\prime}$ $=14$ case, only the highest parallel-resolution run is adequate. The curves from the poorly resolved runs are clearly too flat as a function of $k_{\perp}$ (at low $k_{\perp}$ ). In particular, because they can be several orders of magnitude too high near $k_{\perp}$ $=1$, they may not capture the substantial increase in spectral amplitude which should occur with increasing $k_{\perp}$ (compare the dotted and solid curves for $k_{z}^{\prime}=7$ ).

\section{CONCLUSIONS}

We have shown that for strongly forced RMHD, parallel spectral transfer is self-limiting due to the weakness of the cascade in this direction: There is no need to introduce parallel dissipative terms. As expected, perpendicular spectral transfer is limited in the usual way, i.e., by the dissipation scale in that direction.

These results indicate that RMHD is indeed a selfconsistent model. That is, weakly ${ }^{52}$ non-RMHD fluctuations-either present initially or generated dynamically - are adequately evolved by the RMHD equations, despite the fact that the equations are not formally valid for them. Moreover, since the leading-order couplings for strongly non-RMHD fluctuations are of the resonant hydrolike-nonhydrolike kind (Sec. III B)—associated with 
suppressed parallel transfer but (still) strong perpendicular transfer-one might anticipate that the RMHD equations will also adequately capture the physics associated with these interactions.

One practical conclusion from this study is that in order to correctly simulate the dynamics of the RMHD modes using the RMHD equations, it is necessary to choose both $B_{0}^{\prime}$ and the maximum retained $k_{z}^{\prime}$ large enough so that, in Fourier space, the RMHD region lies inside the computational domain. The required parallel resolution scales as $k_{z}^{\max } \lambda$ $\sim\left(\delta b / B_{0}\right) R_{e}^{1 / 2}$ [see Eqs. (18) and (19)]. The usual restriction of the maximum retained $k_{\perp}$ being greater than the dissipation wavenumber is also required. Simulations based on the RMHD equations should use a domain in which the RMHD region is a well-resolved subset of the computational domain. If this is not done, then the self-limiting of the parallel cascade will not usually be apparent at all $k_{\perp}$. Quantities like the axisymmetric energy spectrum, $E_{\text {axi }}\left(k_{z}^{\prime}, k_{\perp}\right)$, are then likely to be distorted in terms of both spectral shape and spectral amplitude.

Although it is always better to have adequate resolution, one can ask how quantities obtained by integration over one or more directions in $\mathbf{k}$-space, are affected by the parallel resolution. Such quantities include the spectra $E_{z}\left(k_{z}\right)$ and $E_{2 \mathrm{D}}\left(k_{\perp}\right)$, and bulk values like the kinetic energy. As integrated quantities are typically most influenced by the excitation levels of the energy-containing modes, one might hope that they would not be particularly sensitive to the lack of resolution in the parallel direction, since the excitation is very weak at large $k_{z}$. Indeed, energy spectra plots (Fig. 7) indicate that parallel resolution primarily affects the situation at higher $k_{z}$, with relatively weak effects for the lowest $k_{z}$ modes.

We have also discussed the importance of the hydrolike condition to RMHD, and MHD turbulence in general. This condition, or cousins of it, has also been invoked in various other contexts. ${ }^{9,20,22-24}$ These include the definition of the Kubo number, ${ }^{53}$ of relevance in the theory of scattering of particles by MHD turbulence, and the "critical balance" condition, introduced by Higdon ${ }^{34}$ and used by Goldreich and Sridhar $^{13,37}$ (hereafter GS) in their model of strong MHD turbulence. (We note that there are several papers which identify some problems with further conclusions of the GS approach. ${ }^{10,11,54,55}$ )

The critical balance assumption ${ }^{13,37}$ asserts that within the (putative) inertial range of strong MHD turbulence, the k-dependent "nonlinearity parameter," $\chi(\mathbf{k})=k_{\perp} \widetilde{v}_{k} / k_{z} B_{0}$ (in our notation), will dynamically adjust to take values of about unity. As noted in Sec. II, $\chi$ is a special case of $1 / \epsilon_{\mathrm{hydro}}=\tau_{\mathrm{A}}(\mathbf{k}) / \tau_{\mathrm{NL}}(\mathbf{k})$; specifically, when the nonlinear time is approximated using perpendicular components. In effect, substantial anisotropy is being assumed in the definition of $\chi$, whereas this is not the case in the definition of $\epsilon_{\text {hydro }}$. In cases where the anisotropy is strong, the distinction is of course largely irrelevant.

As part of their definition of strong MHD turbulence, GS assume that $\delta u, \delta b \sim B_{0}$. However, as discussed in the body of this paper, it is our view that the "critical balance/ hydrolike condition" style of idea has much wider applica- bility than this. Indeed, whether the turbulence is weak or strong in terms of the size of $\delta b / B_{0}$, we would suggest that there is almost always a region where the turbulence is strong in the sense that the nonlinear interactions are strong. We refer to this as the hydrolike region, defined in Fourier space by the set of Fourier wavevectors whose modes satisfy $\epsilon_{\text {hydro }}(\mathbf{k}) \lesssim 1$, or equivalently $\tau_{\mathrm{NL}}(\mathbf{k}) \lesssim \tau_{\mathrm{A}}(\mathbf{k})$. As discussed in Appendix B, as the mean field is progressively weakened the hydrolike region expands to fill more and more of the (Fourier space) domain.

In closing, we note that in deriving a kinetic equation for weak turbulence, defined by $\epsilon=\delta b / B_{0} \ll 1$, Galtier et al. ${ }^{26}$ observe that "for any turbulence intensity $\epsilon$ there always exists a region of small $k_{\|}$in which the condition $k_{\|} \gg \epsilon^{2} k_{\perp}$ is violated; this corresponds to the nonuniform validity of the kinetic equation." This seems to us to correspond to what we have called the RMHD region, which is inherently strongly nonlinear and not describable using weak turbulence theory. The hydrolike region is the generalization of the RMHD region to the situation where $\epsilon$ is not restricted to be small.

\section{ACKNOWLEDGMENTS}

We would like to express our thanks to David Montgomery and Marco Velli for helpful and informative discussions regarding the RMHD approximation.

This research was supported by grants from the NSF (ATM-9977692 and ATM-0105254), NASA (NAG5-11603), and the New Zealand Marsden Fund (02-UOW-050 MIS).

\section{APPENDIX A: SMALL PARAMETERS AND THE "NATURAL" RMHD UNITS}

Strauss ${ }^{5}$ derivation of the RMHD equations was based upon an expansion in terms of the small parameter $\epsilon_{\text {Strauss }}$ $=l_{\perp} / l_{\|}$, the ratio of characteristic lengthscales in the perpendicular and parallel directions. However, he found that simply expanding in this small parameter was not sufficient; it was also necessary to make some assumptions regarding the ordering of the fluctuation strengths and the timescale. Specifically, $\quad v_{\perp}, b_{\perp} \sim \epsilon_{\text {Strauss }}, \quad B_{z} \sim B_{0}+\epsilon_{\text {Strauss }}^{2}, \quad$ and $\partial / \partial t$ $\sim \epsilon_{\text {Strauss }}$.

In contrast, Montgomery's ${ }^{3}$ derivation was based upon a distinct small parameter $\epsilon_{\text {Mont }}=\delta b / B_{0}$, where $\delta b$ is the rms value for the fluctuating magnetic field and the total field is $\mathbf{B}=\mathbf{B}_{0}+\mathbf{b}(\mathbf{x}, t)$. Again, simply expanding in the small parameter is not sufficient, with additional restrictions on the scalings of other quantities being required. In particular, Montgomery found that the expansion would only remain valid if lengthscales were restricted so that $l_{\perp} \ll l_{\|}$.

The ratio of these two small parameters is

$$
\frac{\epsilon_{\text {Strauss }}}{\epsilon_{\text {Mont }}}=\frac{l_{\perp}}{\delta b} / \frac{l_{\|}}{B_{0}}=\frac{\tau_{\mathrm{NL}}}{\tau_{\mathrm{A}}} \equiv \epsilon_{\text {hydro }},
$$

so that the (global) RMHD condition can be stated as $\epsilon_{\text {Strauss }} \lesssim \epsilon_{\text {Mont }} \ll 1$ (which includes the possibility of $\epsilon_{\text {Strauss }}$ $\left.\ll \epsilon_{\text {Mont }}\right)$. In effect, the Strauss and Montgomery derivations each assumed $\epsilon_{\text {Mont }}=\epsilon_{\text {Strauss }}$. 
TABLE I. Various subsets of the full set of 3D MHD modes, specified by reference to their Fourier modes. A dc field $\mathbf{B}_{0}=\hat{\mathbf{z}} B_{0}$ is assumed to be present with its direction defining the parallel coordinate. The $2 \mathrm{D}$ planes have $\hat{\mathbf{z}}$ as their normal.

\begin{tabular}{lccc}
\hline \hline $\begin{array}{l}\text { Name of } \\
\text { subset }\end{array}$ & $\begin{array}{c}\text { Geometry of } \mathbf{v} \\
\text { (and } \mathbf{b})\end{array}$ & $\begin{array}{c}\text { Domain and } \\
\text { range }\end{array}$ & Constraints \\
\hline $3 \mathrm{D}$ & $\mathbf{v}\left(\mathbf{k}_{\perp}, k_{\|}\right)=(u, v, w)$ & $f: \mathrm{R}^{3} \rightarrow \mathrm{R}^{3}$ & $\ldots$ \\
$2 \mathrm{D}$ & $\mathbf{v}_{\perp}\left(\mathbf{k}_{\perp}, 0\right)=(u, v, 0)$ & $f: \mathrm{R}^{2} \rightarrow \mathrm{R}^{2}$ & $\partial / \partial z=0$, \\
& $\mathbf{v}\left(\mathbf{k}_{\perp}, 0\right)=(u, v, w)$ & $f: \mathbb{R}^{2} \rightarrow \mathbb{R}^{3}$ & $\hat{\mathbf{z}} \cdot \mathbf{v}=0$. \\
$2 \frac{1}{2} \mathrm{D}$ & $\mathbf{v}\left(\mathbf{k}_{\perp}, k_{\|}^{\text {slow }}\right)=(u, v, 0)$ & $f: \mathbb{R}^{2} \times \mathbb{R}^{*} \rightarrow \mathbb{R}^{2}$ & $\partial / \partial z=0$ \\
$\mathrm{RMHD}^{\mathrm{a}}$ & & & $\tau_{\mathrm{NL}}(\mathbf{k}) \leqslant \tau_{\mathrm{A}}(\mathbf{k})$, \\
& $v_{\perp}, v_{\|} \ll B_{0}$. \\
hydrolike & $\mathbf{v}\left(\mathbf{k}_{\perp}, k_{\|}\right)=(u, v, w)$ & $f: \mathbb{R}^{3} \rightarrow \mathbb{R}^{3}$ & $\tau_{\mathrm{NL}}(\mathbf{k}) \leqslant \tau_{\mathrm{A}}(\mathbf{k})$ \\
\hline \hline
\end{tabular}

${ }^{a}$ RMHD also includes parallel components of $\mathbf{v}$ and $\mathbf{b}$, in which case one has $f: \mathbb{R}^{2} \times \mathbb{R}^{*} \rightarrow \mathbb{R}^{3}$, where $\mathbb{R}^{*}$ denotes a subset of $\mathrm{R}$ centered around zero (the set of $k_{\|} \mathrm{s}$ ). However, these components are dynamically passive and often ignored (Refs. 3 and 6).

${ }^{\mathrm{b}}$ Also called quasi- $2 D$ when $B_{0}$ is large.

An alternative derivation of the RMHD equations can be accomplished by expanding the full 3D MHD equations perturbatively with respect to both of these parameters (treated as independent), where

$$
\begin{aligned}
& \mathbf{B}=\frac{B_{0}^{\prime} \hat{\mathbf{z}}}{\epsilon_{\text {Mont }}}+\mathbf{b}^{\prime}+\epsilon_{\text {Mont }} \mathbf{b}_{1}^{\prime}+\ldots, \\
& z=\frac{z^{\prime}}{\epsilon_{\text {Strauss }}}+z_{0}^{\prime}+\epsilon_{\text {Strauss }} z_{1}^{\prime}+\ldots,
\end{aligned}
$$

and a prime (') denotes the values in the rescaled (natural) units. One finds that the $B_{0}^{\prime} \partial j / \partial z^{\prime}$ term in Eq. (1) above [cf. Eq. (3)] should really be written

$$
\left(\frac{B_{0}^{\prime}}{\epsilon_{\text {Mont }}}\right)\left(\epsilon_{\text {Strauss }} \frac{\partial}{\partial z^{\prime}}\right) j
$$

where $j=\hat{\mathbf{z}} \cdot \boldsymbol{\nabla} \times \mathbf{b}^{\prime}$. The situation is similar for the analogous term in the equation for the vector potential. These forms make it explicit that although $z^{\prime}$ and $B_{0}^{\prime}$ are $O(1)$, the actual physical quantities associated with these "natural units" variables are both large.

In the simulations discussed in Sec. IV the RMHD equations are solved for values of $B_{0}^{\prime \prime}=B_{0}^{\prime} \epsilon_{\text {Strauss }} / \epsilon_{\text {Mont }}$ ranging from $1 / 2$ to 8 . (Note that $B_{0}^{\prime \prime}$ is denoted as $B_{0}^{\prime}$ outside of this Appendix.) In the context of the investigation discussed there, an appropriate interpretation for the physical fields is to consider $\epsilon_{\text {Strauss }}$ to be fixed, independent of the mean field strength, so that for all simulations of a given parallel resolution the same set of (physical) $k_{\|}$'s is employed. Different values of $B_{0}^{\prime \prime}$ then represent changes in the physical $B_{0}$, as desired.

\section{APPENDIX B: SUBSETS OF INCOMPRESSIBLE MHD}

Various subsets of full incompressible 3D MHD (with a $\mathrm{dc}$ field present) are of interest and importance in the context of MHD turbulence and its applications. Table I lists some defining properties for several such subsets. The intersections of the various subsets are depicted in Fig. 8 using a Venn diagram.

A key distinction to be made is that between the hydrolike modes, defined as the set of modes which satisfy $\tau_{\mathrm{NL}}(\mathbf{k}) \lessgtr \tau_{\mathrm{A}}(\mathbf{k})$, and the RMHD modes which must, in addition, satisfy the restriction that the fluctuations have small amplitudes, e.g., $\delta b / B_{0} \ll 1$. As discussed throughout this paper, satisfaction of both of these constraints requires also that the parallel lengthscales are long compared to the perpendicular ones. Said differently, the RMHD modes form a proper subset of the hydrolike modes.

Clearly not all of the $2 \mathrm{D}$ and $2 \frac{1}{2} \mathrm{D}$ modes present in a system are necessarily RMHD in character (Fig. 8), since the small amplitude restriction must also be satisfied. This is to be contrasted with the situation for the hydrolike modes, for which the $2 \mathrm{D}$ and $2 \frac{1}{2} \mathrm{D}$ modes are proper subsets. Moreover, the strictly $2 \frac{1}{2} \mathrm{D}$ modes which are also RMHD modes must have nonzero parallel components, since otherwise they would be pure 2D modes; that is, in this case account must be taken of $v_{\|}(\mathbf{k})$ and $b_{\|}(\mathbf{k})$, even though they are dynamically passive. ${ }^{3,6}$

In circumstances where there is a strong mean magnetic

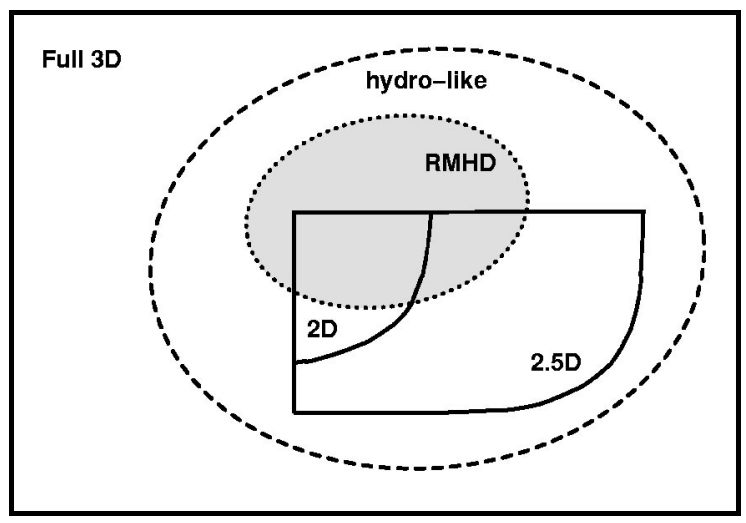

FIG. 8. Venn diagram of some (modal) subsets of incompressible 3D MHD turbulence. The boundaries for the RMHD subset and the hydrolike subset (shown as broken curves) depend upon the magnitude of $\mathbf{B}_{0}$. 
field, satisfaction of the hydrolike condition for a mode would typically require that $k_{\perp} \gg k_{\|}$, so that these modes are then conveniently referred to as quasi-2D. On the other hand, as $B_{0} \rightarrow 0$ the geometry of $\mathbf{k}$ becomes of less relevance in satisfying the defining timescale restriction, and so the term quasi-2D becomes increasingly misleading. Whatever term is used to describe such modes, the key point is that as far as dynamical processes are concerned wave effects are usually of secondary importance. (Although exceptions are possible; for example, within an RMHD model counter-propagating waves can be used to drive the turbulence..$^{50,56,57}$ )

Finally, we note that the definition of the hydrolike modes could be generalized somewhat by using a $\tau_{\mathrm{A}}(\mathbf{k})$ based upon the (local) mean field as felt by that $\mathbf{k}$, rather than just the global mean field, e.g., $\mathbf{B}_{\text {local }}=\mathbf{B}_{0}+\langle\mathbf{b}\rangle_{\text {local }}$. This would presumably be in better accord with the local physics and any wave-like attributes characteristic of the fluctuations. ${ }^{24,58,59}$

\section{APPENDIX C: RMHD AND NONPERIODIC BOUNDARY CONDITIONS}

Cases with nonperiodic boundary conditions (e.g., finite length nonperiodic domains) require some care in defining the Alfvénic timescales. Indeed, there are now two such timescales to consider. The first is the usual wave period $\tau_{\mathrm{A}}=2 \pi /\left|\mathbf{k} \cdot \mathbf{B}_{0}\right|$, which is the time taken to propagate one wavelength, essentially the same as Eq. (4). The second is the box-crossing time, $\tau_{\text {box }}=L_{z} / B_{0}$, which is the time it takes an Alfvén wave to propagate across the domain. For periodic boundary conditions, it is not possible to consider fluctuations with wavelengths longer than the box length, hence $\tau_{\mathrm{A}} \leqslant \tau_{\text {box }}$. In the nonperiodic case, however, the wavevectors $\mathbf{k}$ are unrestricted. In some circumstances the effective wavelength of some $\mathbf{k}$ vectors can be very large, leading to $\tau_{\mathrm{A}} \gg \tau_{\mathrm{box}}$. In solar physics, for example, the footpoint motions of magnetic field-lines which emerge through the photosphere and extend upwards into the corona are often quasi-static. Thus, when considering a 'box' of coronal plasma of finite height, the slowness of the field-line meanderings can easily lead to $\tau_{\mathrm{A}} \gg \tau_{\text {box }}$.

As far as the RMHD condition is concerned $\tau_{\text {box }}$ is of no direct relevance, and thus might be thought to play no role in determining whether RMHD is an appropriate approximation to use in a given system. However, the situation is complicated by the presence of other important timescales associated with effects like forcing and/or reflection of waves. It transpires that the value of $\tau_{\text {box }}$ compared to the other timescales in the system is important, and may determine energy transfer rates and spectra. ${ }^{18,56,60}$

${ }^{1}$ B. B. Kadomtsev and O. P. Pogutse, Sov. Phys. JETP 38, 283 (1974).

${ }^{2}$ S. E. Kruger, C. C. Hegna, and J. D. Callen, Phys. Plasmas 5, 4169 (1998).

${ }^{3}$ D. C. Montgomery, Phys. Scr., T T2/1, 83 (1982).

${ }^{4}$ P. J. Morrison and R. P. Hazeltine, Phys. Fluids 27, 886 (1984).

${ }^{5}$ H. R. Strauss, Phys. Fluids 19, 134 (1976).

${ }^{6}$ G. P. Zank and W. H. Matthaeus, J. Plasma Phys. 48, 85 (1992).

${ }^{7}$ D. O. Gómez, P. Dmitruk, and L. J. Milano, Sol. Phys. 195, 299 (2000).

${ }^{8}$ S. Oughton, P. Dmitruk, and W. H. Matthaeus, Coronal heating and reduced MHD, in Turbulence and Magnetic Fields in Astrophysics, edited by
E. Falgarone and T. Passot (Springer, New York, 2003), Vol. 614, pp. $28-55$.

${ }^{9}$ R. Kinney and J. C. McWilliams, Phys. Rev. E 57, 7111 (1998),

${ }^{10}$ C. S. Ng and A. Bhattacharjee, Astrophys. J. 465, 845 (1996).

${ }^{11}$ C. S. Ng and A. Bhattacharjee, Phys. Plasmas 4, 605 (1997).

${ }^{12}$ S. Oughton, S. Ghosh, and W. H. Matthaeus, Phys. Plasmas 5, 4235 (1998).

${ }^{13}$ P. Goldreich and S. Sridhar, Astrophys. J. 438, 763 (1995).

${ }^{14}$ K. Nakayama, Astrophys. J. 556, 1027 (2001).

${ }^{15}$ See Zank and Matthaeus (Ref. 6) for discussion of difficulties associated with derivations based on the incompressible MHD equations.

${ }^{16}$ D. Fyfe, G. Joyce, and D. Montgomery, J. Plasma Phys. 17, 317 (1977).

${ }^{17}$ R. H. Kraichnan, Phys. Fluids 10, 1417 (1967).

${ }^{18}$ P. Dmitruk, D. O. Gómez, and W. H. Matthaeus, Phys. Plasmas 10, 3584 (2003).

${ }^{19}$ Goldreich and Sridhar (Refs. 13 and 37) term the reciprocal of $\epsilon_{\text {hydro }}$, evaluated using perpendicular values in $\tau_{\mathrm{NL}}(\mathbf{k})$, the "Nonlinearity parameter." See also Sec. V.

${ }^{20}$ S. R. Cranmer and A. A. van Ballegooijen, Astrophys. J. 594, 573 (2003).

${ }^{21}$ W. H. Matthaeus, S. Oughton, S. Ghosh, and M. Hossain, Phys. Rev. Lett. 81, 2056 (1998).

${ }^{22}$ B. J. Vasquez and J. V. Hollweg, "Nonlinear Alfvén waves. I. Interactions between outgoing and ingoing Alfvén waves according to an amplitude expansion," J. Geophys. Res. (to be published).

${ }^{23}$ B. J. Vasquez, S. A. Markovskii, and J. V. Hollweg, "Nonlinear Alfvén waves. II. The influence of wave advection and finite wavelength effects," J. Geophys. Res. (to be published).

${ }^{24}$ J. Cho and E. T. Vishniac, Astrophys. J. 539, 273 (2000).

${ }^{25}$ S. Galtier, S. V. Nazarenko, A. C. Newell, and A. Pouquet, J. Plasma Phys. 63, 447 (2000).

${ }^{26}$ S. Galtier, S. V. Nazarenko, A. C. Newell, and A. Pouquet, Astrophys. J. Lett. 564, L49 (2002).

${ }^{27}$ R. Kinney and J. C. McWilliams, J. Plasma Phys. 57, 73 (1997).

${ }^{28}$ A. Bondeson, Phys. Fluids 28, 2406 (1985).

${ }^{29}$ R. Grappin, Phys. Fluids 29, 2433 (1986).

${ }^{30}$ S. Oughton, E. R. Priest, and W. H. Matthaeus, J. Fluid Mech. 280, 95 (1994).

${ }^{31}$ J. V. Shebalin, W. H. Matthaeus, and D. Montgomery, J. Plasma Phys. 29, 525 (1983).

${ }^{32}$ The existence of such a boundary seems to have first been mooted by Montgomery and Turner (Ref. 33) (p. 828): "Real life is also likely to involve a transition region, with $\mathbf{k} \cdot \mathbf{B}_{0}$ small but nonzero, where linear and nonlinear effects will be of comparable magnitude. The direction of flow of excitations in $\mathbf{k}$-space across this transition region is one of the major unanswered questions remaining."

${ }^{33}$ D. C. Montgomery and L. Turner, Phys. Fluids 24, 825 (1981).

${ }^{34}$ J. C. Higdon, Astrophys. J. 285, 109 (1984).

${ }^{35}$ A. Bhattacharjee and C. S. Ng, Astrophys. J. 548, 318 (2001).

${ }^{36}$ V. Carbone and P. Veltri, Geophys. Fluid Dyn. 52, 153 (1990).

${ }^{37}$ P. Goldreich and S. Sridhar, Astrophys. J. 485, 680 (1997).

${ }^{38}$ J. Maron and P. Goldreich, Astrophys. J. 554, 1175 (2001).

${ }^{39}$ W. H. Matthaeus, S. Ghosh, S. Oughton, and D. A. Roberts, J. Geophys. Res. 101, 7619 (1996).

${ }^{40}$ S. Sridhar and P. Goldreich, Astrophys. J. 432, 612 (1994).

${ }^{41}$ Note that there are difficulties associated with wavepacket decompositions of space-filling turbulence. For example, although there is an aspect of super-position connected with the decomposition, it cannot be a linear super-position since turbulence is nonlinear. Additionally, wavepackets should typically be spatially well-separated, and typically maintain at least some semblance of coherence after two of them interact. We are not aware of any work in which such wavepacket behavior has been identified or tracked.

${ }^{42}$ From Eq. (10), the 2D modes are always resonant since by definition they have $p_{\|}=q_{\|}=0$. This is sometimes refered to as "trivial resonance."

${ }^{43}$ Provided boundary conditions allow. See Appendix C and Ref. 56.

${ }^{44}$ P. S. Iroshnikov, Astron. Zh. 40, 742 (1963) [Sov. Astron 7, 566 (1964)].

${ }^{45}$ R. H. Kraichnan, Phys. Fluids 8, 1385 (1965).

${ }^{46}$ W. H. Matthaeus and Y. Zhou, Phys. Fluids B 1, 1929 (1989).

${ }^{47}$ D. C. Montgomery, Lecture Notes on Turbulence (World Scientific, Singapore, 1989), p. 75, Lecture Notes from the NCAR-GTP Summer School, June 1987. 
${ }^{48}$ Note that the quasi-resonance condition given as Eq. (11) is not applicable for $H-H$ interactions since for these modes the amplitudes $A^{ \pm}$cannot be treated as slowly varying.

${ }^{49}$ However, perpendicular transfer can eventually move the excitation back inside the hydrolike region if $k_{\text {diss }}$ is sufficiently large [Fig. 1(b)].

${ }^{50} \mathrm{~S}$. Oughton, W. H. Matthaeus, P. Dmitruk, L. M. Milano, G. P. Zank, and D. J. Mullan, Astrophys. J. 551, 565 (2001).

${ }^{51}$ That is, $\Delta \mathbf{A}_{k}^{-}$summed over $\mathbf{p}, \mathbf{q}$ subject to $\mathbf{k}=\mathbf{p}+\mathbf{q}$.

${ }^{52}$ Here, weakly non-RMHD could be defined as $\mathbf{v}$ and $\mathbf{b}$ still being smallamplitude and plane-polarized, but having $\tau_{\mathrm{NL}}(\mathbf{k})$ larger than $\tau_{\mathrm{A}}(\mathbf{k})$, although not too much so.

${ }^{53}$ R. Kubo, J. Math. Phys. 4, 174 (1963).
${ }^{54}$ D. C. Montgomery and W. H. Matthaeus, Astrophys. J. 447, 706 (1995).

${ }^{55}$ S. V. Nazarenko, A. C. Newell, and S. Galtier, Physica D 152-153, 646 (2001).

${ }^{56}$ P. Dmitruk, W. H. Matthaeus, L. J. Milano, and S. Oughton, Phys. Plasmas 8, 2377 (2001).

${ }^{57}$ W. H. Matthaeus, G. P. Zank, S. Oughton, D. J. Mullan, and P. Dmitruk, Astrophys. J. Lett. 523, L93 (1999).

${ }^{58}$ L. J. Milano, W. H. Matthaeus, and P. Dmitruk, Phys. Plasmas 10, 2287 (2003).

${ }^{59}$ L. J. Milano, W. H. Matthaeus, P. Dmitruk, and D. C. Montgomery, Phys. Plasmas 8, 2673 (2001).

${ }^{60}$ P. Dmitruk and W. H. Matthaeus, Astrophys. J. 597, 1097 (2003). 